\title{
TSPC Flip-Flop Circuit Design with Three-Independent-Gate Silicon Nanowire FETs
}

\author{
Xifan Tang ${ }^{1}$, Jian Zhang ${ }^{2}$, Pierre-Emmanuel Gaillardon ${ }^{2}$, Giovanni De Micheli ${ }^{2}$ \\ ${ }^{1}$ School of Computer and Communication Sciences, EPFL, Switzerland \\ ${ }^{2}$ Integrated Systems Laboratory, EPFL, Switzerland \\ Email: xifan.tang@epfl.ch
}

\begin{abstract}
True Single-Phase Clock (TSPC) Flip-Flops, based on dynamic logic implementation, are area-saving and highspeed compared to standard static flip-flops. Furthermore, logic gates can be embedded into TSPC flip-flops which significantly improves performance. As a promising approach to keep the pace of Moore's Law, functionality-enhanced devices with multiple independent gates have drown many recent interests. In particular Three-Independent-Gate Silicon Nanowire FETs (TIG SiNWFETs) can realize the functionality of two serial transistors in a single device. Therefore, they open new opportunities to compact designs in both arithmetic and control circuits. In this paper, we propose TSPC flip-flop implementation with asynchronous set and reset using the compactness of TIG SiNWFET. Electrical simulations show that TIG SiNWFET-based TSPC flip-flop improves nearly $20 \%, 30 \%$ and $7 \%$ in area, delay and leakage power respectively as compared to its LSTP FinFET counterpart at $22 \mathrm{~nm}$.
\end{abstract}

\section{INTRODUCTION}

As an essential component of sequential circuits, flipflops have a large impact on the area, speed and power consumption of modern digital circuits [1]. Flip-flops can be built using either static or dynamic design [2]. Static flip-flops are usually based around two inverter-based latches, that have a large impact on area and delay. However, flip-flops based on dynamic logic have advantages in high operating speed and area density compared to static ones [1]. Most flip-flops require both clock signal and its inversion, which challenges clock tree synthesis. However, True Single-Phase Clock (TSPC) flipflop [3], a dynamic flip-flop, needs only a single clock signal. Using single phase clock and dynamic logic do not only lead to compact design but also faster response. In addition, a unique feature of TSPC flip-flop is the reduction of the overall setup time and delay obtained by embedding logic gates inside the structure [2]. For these reasons, TSPC flip-flop is widely used in many high-speed applications [4].

In order to extend Moore's Law, intensive researches on emerging technologies, such as carbon nanotubes [5], graphene [6], and SiNWFET [7] recently proposed novel devices that have better channel controllability and functionality. Among them, SiNWFET is promising thanks to its controllable ambipolar behavior [9] [7], i.e., the dynamic reconfiguration of the device polarity from $n$ - to $p$-type thanks to an extra gate terminal, and its CMOS compatible fabrication process. Recent researches [9] [10] present efficiencies of Double-IndependentGate (DIG) and Three-Independent-Gate (TIG) SiNWFETs in implementing combinational circuits, in terms of area and delay, due to the design compactness brought by the devices. However, only limited investigations has been done so far on sequential elements.

In this paper, we propose to combine the performance of TSPC flip-flop design with the compactness offered by
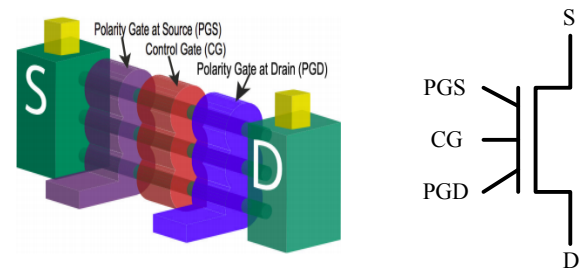

(a)

(b)

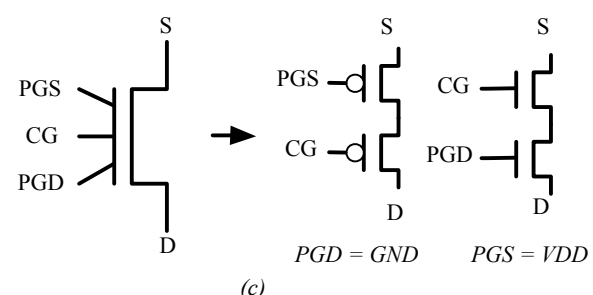

Fig. 1. TIG SiNWFET structure (a), symbol (b) and polarity control (c)

TIG SiNWFETs. A TIG SiNWFET-based TSPC flip-flop with asynchronous set and reset is therefore presented. Performance evaluation obtained by electrical simulations demonstrates an area, delay and leakage power reduction of almost $20 \%, 30 \%$ and $7 \%$ respectively. TSPC flip-flop with logic gate embedded is investigated as well. For AND gate, TIG SiNWFET implementation presents a $21 \%$ area gain, a $6 \%$ speed up and $45 \%$ leakage reduction.

The rest of the paper is organized as follows. In Section II, basics of TIG SiNWFET and CMOS TSPC flip-flop are introduced. In Section III, TIG SiNWFET TSPC flip-flop circuit design and electrical characterizations are presented. In Section IV, XOR embedded TIG SiNWFET TSPC flip-flop is introduced. In Section V, conclusions are drawn.

\section{BACKGROUND}

In this section, operation of TIG SiNWFET are briefly introduced. Then, the classical CMOS design of TSPC flipflop and its logic-gate-embedded feature are reviewed.

\section{A. TIG SiNWFET}

As shown in Fig. 1, TIG SiNWFET [9] is a verticallystacked silicon nanowire FET with three independent gate-allaround regions. Two independent polarity gates, Polarity Gate at Source (PGS) and Polarity Gate at Drain (PGD) respectively control the tunneling of electrons and holes, while the Control 


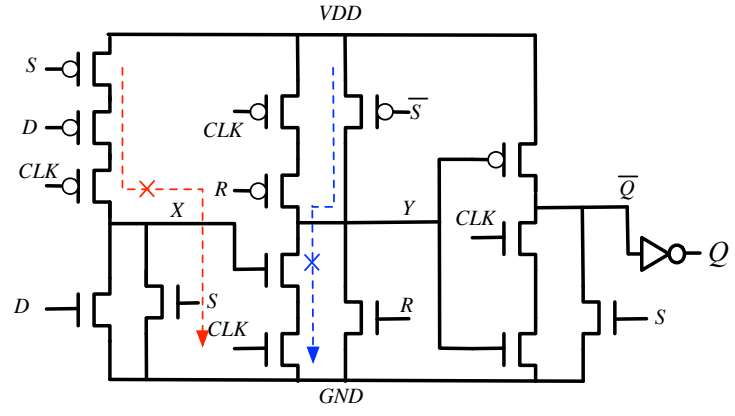

Fig. 2. CMOS TSPC flip-flop with set and reset [3] [14]

Gate (CG) switches the channel conduction as in conventional MOSFETs. As shown in Fig. 1, TIG SiNWFET can equivalently realize two serial transistors with a unique device, by setting PGS to VDD (for two serial $n$-type transistors) or PGD to GND (for two serial $p$-type transistors). In traditional CMOS design style, replacing two serial transistors by a unique TIG SiNWFET leads to about $33 \%$ area saving though the area of a TIG SiNWFET is $50 \%$ larger than a MOSFET. Note that two serial transistors frequently take place not only in combinational gates, such as XOR, NAND, and AOI, but also in dynamic logic, such as TSPC flip-flop. Hence, by applying conversion in Fig. 1, area savings are expected. Additionally, circuits can be speed up as a single TIG SiNWFET presents less internal capacitance than two serial CMOS transistors.

\section{B. CMOS TSPC Design}

CMOS TSPC flip-flop can be built with only 9 transistors, which is very compact as compared to static version with 22 transistors [2]. A TSPC flip-flops with asynchronous reset and set requires 6 additional transistors for pulling-up to VDD or pulling-down to GND at each stage.

As depicted in Fig. 2, CMOS TSPC flip-flop is composed of three stages.

1) $1^{\text {st }}$ Stage - Precharge Stage, CLK-Low Enable: The first stage is a precharge stage. The stage is transparent when $C L K=0$, i.e. the node $X$ is updated with the value of $D$ only when $\mathrm{CLK}=0$.

2) $2^{\text {nd }}$ Stage - Latch Stage: The second stage is a latch stage, storing the value of node $\mathrm{X}$ at the rising edge of CLK. When $C L K=0$, node $Y$ is precharged to VDD. At the rising edge of CLK, node $\mathrm{Y}$ can be updated to GND if node $\mathrm{X}=1$. Note that even if node $X$ can be pulled-down when $C L K=1$, the latched value at node $\mathrm{Y}$ cannot be modified.

3) $3^{\text {rd }}$ Stage - Precharge Stage, CLK-High Enable: The third stage is another precharge stage but is transparent when $C L K=1$. The third stage propagates node $\mathrm{Y}$ only when $\mathrm{CLK}=1$.

However, based on precharging and discharging, TSPC flip-flop depends on internal capacitance for storage, which requires periodical refreshing (on the order of milliseconds [2]).

In addition, logic gates can be directly inserted into the first stage of CMOS TSPC flip-flop. Fig. 3 depicts a TSPC flip-flop with a prior AND function. The setup time of a single TSPC flip-flop increases but considering a AND gate cascaded by a standard TSPC flip-flop, the overall setup time decreases [2].

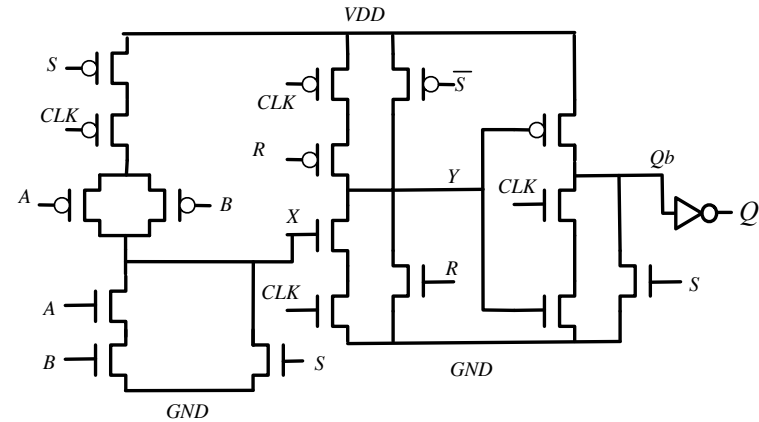

Fig. 3. CMOS AND-gate embedded TSPC schematic

\section{STANDARD TIG SINWFET TSPC FLIP-FLOP}

In this section, TIG SiNWFET-based TSPC flip-flop design is proposed. First, we discuss the circuit structure. Second, the functionality of the TIG SiNWFET TSPC flip-flop is verified by electrical simulations, and comparison in area and delay between standard CMOS and TIG SiNWFET designs is discussed.

\section{A. TSPC Flip-flop Structural Modifications}

As introduced in Section II, TIG SiNWFET leads to area and timing efficiency in realizing two serial in a unique device. This situation frequently takes place in CMOS TSPC flipflop operations (Fig. 2). In addition, TIG SiNWFET can be dynamically reconfigured from $n$ - to $p$-type [9], depending on the signal applied to its gates. Therefore, it is possible to dynamically convert a pull-up transistor into a pull-down one and vice-versa. A combination of these two properties is used to compact the original TSPC flip-flop design.

Fig. 4 depicts a TIG SiNWFET TSPC flip-flop with asynchronous set and reset operations (High Enabled). The novel circuit consists of only 8 TIG SiNWFETs and its schematic fits the layout style in [10], consuming only 3 tiles. By applying design rules of Fig. 1, all control gates and polarity gates are fully used. Compared with CMOS design (Fig. 2), the novel circuit removes the pull-up and pull-down transistors at first stage and third stage. Transistor N1 (highlighted by a red rectangle in Fig. 4) plays a dual role, as it replaces part of pull-up and pull-down branches of the first stage of Fig. 2. Transistor $\mathbf{N} 2$ plays an identical role for the third stage. In addition to merge serial transistors into single devices, improvements are done at the two pre-charge stages.

1) Improvements at $1^{\text {st }}$ Stage: Asynchronous set requires node $\mathrm{X}$ to be pulled down to GND at the first stage even when $\mathrm{CLK}=0$ and $\mathrm{D}=0$. In CMOS design, an additional pulldown transistor, controlled by signal $\mathrm{S}$, is required in order to avoid a path from VDD to GND (blue dashed line in Fig. 2) when $C L K=1$ and $D=0$. Then, to prevent another path from VDD to GND (red dashed line at first stage in Fig. 2) when $\mathrm{CLK}=0$ and $\mathrm{D}=0$, a pull-up transistor, controlled again by signal $\mathrm{S}$, is added. The transistor N1 in Fig. 4 realizes this dual functionality. When set is enabled, transistor N1 switches from $n$-type to $p$-type, while its source is set to VDD. Thus, transistor $\mathrm{N} 1$ is only in on state when $\mathrm{CLK}=0$ and $\mathrm{X}=0$ (when $\mathrm{D}=0, \mathrm{X}=0$ ), which ensures that node $\mathrm{Y}$ can be pulled up to VDD whatever CLK and D are. 


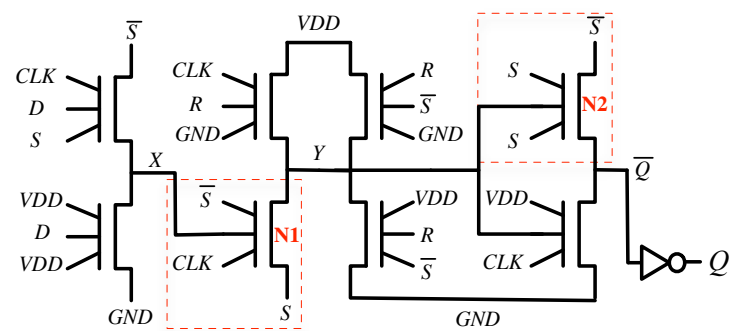

Fig. 4. TIG SiNWFET TSPC schematic

2) Improvements at $3^{\text {rd }}$ Stage: Asynchronous set requires immediate logic-high output when enabled. Therefore, the third stage should be pulled down once set is enabled even when CLK $=0$. In CMOS design, a pull-down transistor is required. However, in TIG SiNWFET design, similarly, transistor N2 switches from $p$-type to $n$-type, while its source is set to GND when set is enabled. As node $\mathrm{Y}$ is pulled up to VDD when set is enabled, transistor $\mathbf{N} 2$ is in on state, thereby pulling down node $\overline{\mathrm{Q}}$ to GND.

\section{B. Transient Validation}

To validate the correct behavior of the cell under asynchronous set and reset, electrical simulations are run and transient waveforms are shown in Fig. 5 and Fig. 6. A simple 22nm TIG SiNWFET table-based compact model, derived from [9], is used with HSPICE simulator. Fig. 5 verifies that the output $\mathrm{Q}$ can be pulled up once set is enabled even during the most challenging case $(\mathrm{CLK}=0, \mathrm{D}=0$ and $\mathrm{Q}=0)$. For asynchronous reset, the most challenging case happens when $\mathrm{CLK}=0, \mathrm{D}=1$ and $\mathrm{Q}=1$. From Fig. 6, the output $\mathrm{Q}$ is observed to be correctly pulled down, when set operation is triggered. Once set/reset signals are de-asserted, the output Q switches again accordingly to the next clock rising edge.

\section{Circuit-level Performance Results}

To evaluate the performance of our flip-flop design, we consider fours major metrics: area, setup time, hold time, and clock to Q delay. In this section, experimental results, obtained

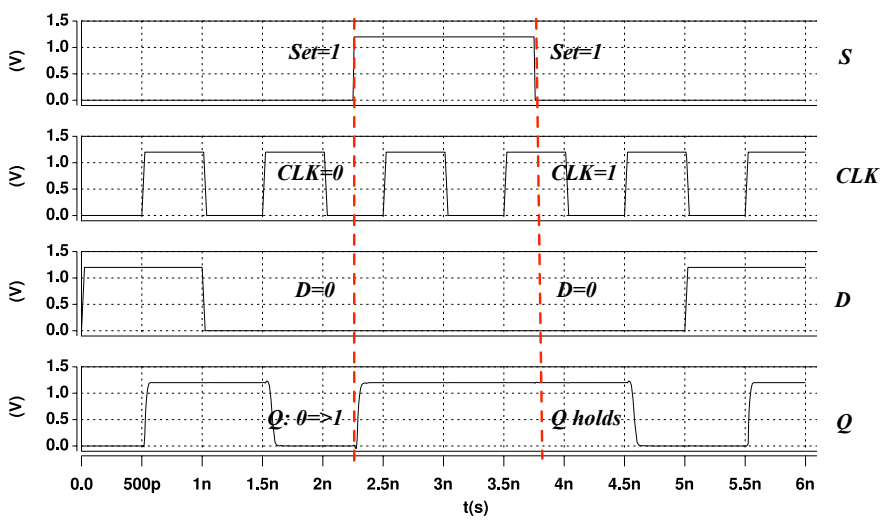

Fig. 5. TIG SiNWFET TSPC transient simulation for asynchronous set
TABLE I. Comparison between FinFET and TIG SiNWFET TSPC flip-flops

\begin{tabular}{|c|c|c|c|c|}
\hline \multicolumn{2}{|c|}{ Benchmark/TSPC } & $\begin{array}{c}\text { LSTP } \\
\text { FinFET }\end{array}$ & $\begin{array}{c}\text { TIG } \\
\text { SiNWFET }\end{array}$ & Comparison \\
\hline \multicolumn{2}{|c|}{ Area(\# of transistors) } & 15 & 12 & $-20.00 \%$ \\
\hline \multirow{3}{*}{ Setup Time } & Rise (ps) & -110.13 & -109.22 & $0.83 \%$ \\
\hline & Fall (ps) & -79.55 & -81.75 & $-2.77 \%$ \\
\hline & Average (ps) & -94.84 & -95.5 & $-0.68 \%$ \\
\hline \multirow{3}{*}{ Hold Time } & Rise (ps) & 86.03 & 86.90 & $1.01 \%$ \\
\hline & Fall (ps) & 117.46 & 114.5 & $-2.52 \%$ \\
\hline & Average (ps) & 101.75 & 100.7 & $-1.03 \%$ \\
\hline \multirow{3}{*}{ Clock To Q Delay } & Rise (ps) & 22.22 & 13.42 & $-39.60 \%$ \\
\hline & Fall (ps) & 34.88 & 25.35 & $-27.32 \%$ \\
\hline & Average (ps) & 28.55 & 19.39 & $-32.10 \%$ \\
\hline \multicolumn{2}{|c|}{ Leakage Power(nW) } & 0.26 & 0.24 & $-7.00 \%$ \\
\hline
\end{tabular}

by electrical simulations, are compared between TSPC flipflop designs, implemented both in traditional CMOS (Fig. 2) and in TIG SiNWFET (Fig. 4), using a technological node of $22 \mathrm{~nm}$. Note that static flip-flops are out of the scope of this paper and therefore are not discussed. For CMOS technology, we use PTM 22nm LSTP FinFET model [12]. To accurately measure the minimum setup time/hold time and clock to $\mathrm{Q}$ delay, a binary search approach is used by setting a delay tolerance corresponding to $10 \%$ of the reference delay and a resolution of $0.01 \mathrm{ps}$ [11]

Table I shows the comparison the two TSPC flip-flop implementations. Thanks to the compactness properties of TIG SiNWFETs, an area saving of up to $20 \%$ is achievable. Regarding timing performances, TIG SiNWFET TSPC flip-flop reduces its internal delay by $30 \%$ on average. The remarkable performance gains come from the area reduction given by TIG SiNWFETm as well as the intrinsic parasitic capacitance reduction given by a unique device instead of two serial CMOS transistors.

\section{Logic Gates Embedded TIG SiNWFET TSPC FLIP-FLOP}

In addition to the realization of more compact pull-up and pull-down networks, TIG SiNWFETs are also able to implement the AND logic function natively [9]. This feature can be efficiently embedded into TIG SiNWFET flip-flop.

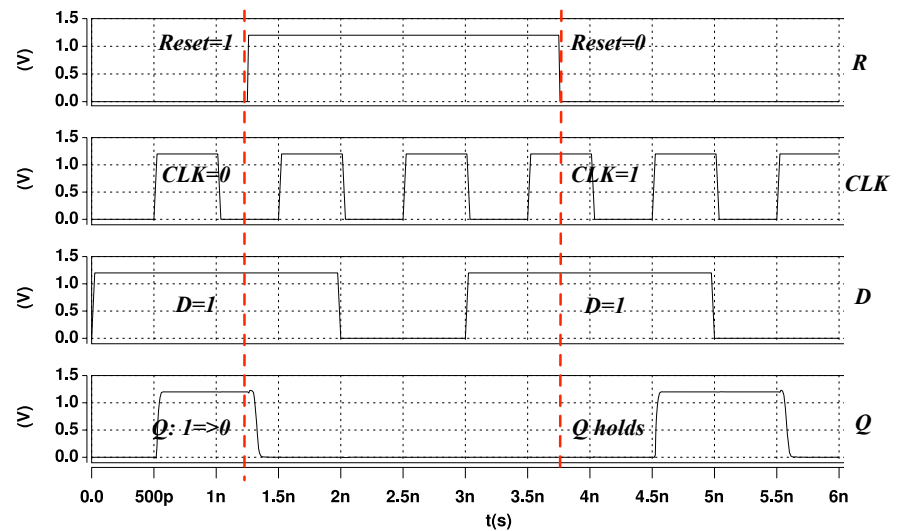

Fig. 6. TIG SiNWFET TSPC transient simulation for asynchronous reset 


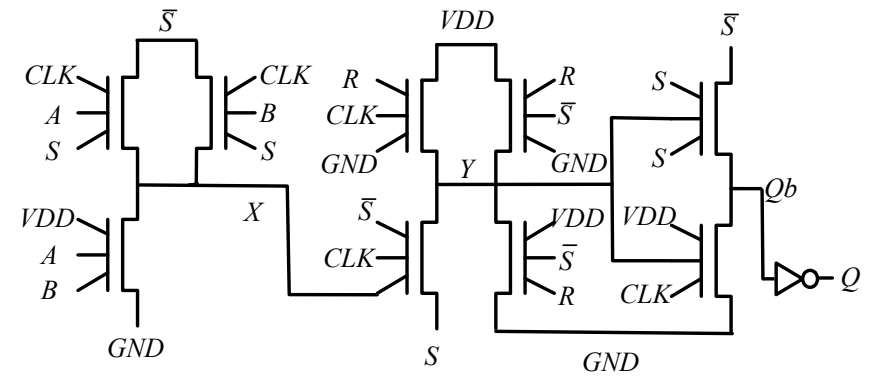

Fig. 7. TIG SiNWFET AND-gate embedded TSPC schematic

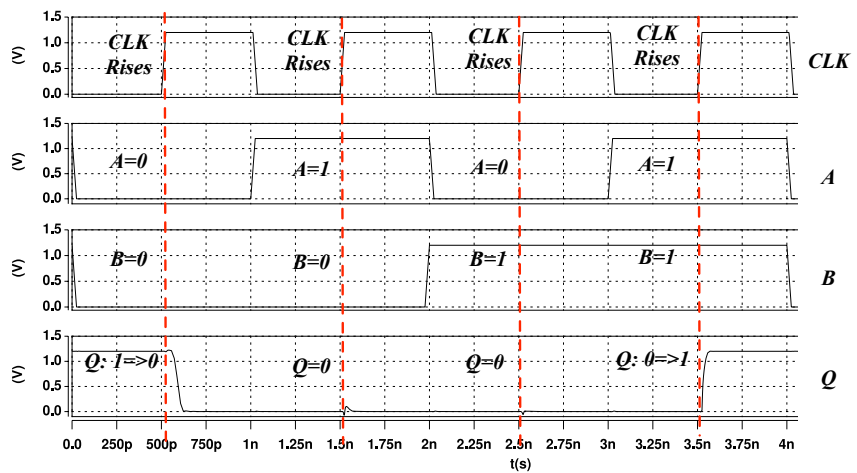

Fig. 8. TIG SiNWFET AND-gate embedded TSPC transient simulation

TABLE II. Comparison between LSTP FinFET and TIG SiNWFET TSPC flip-flops

\begin{tabular}{|c|c|c|c|c|}
\hline \multicolumn{2}{|c|}{ Benchmark/TSPC AND } & $\begin{array}{c}\text { LSTP } \\
\text { FinFET }\end{array}$ & $\begin{array}{c}\text { TIG } \\
\text { SiNWFET }\end{array}$ & Comparison \\
\hline \multicolumn{2}{|c|}{ Area(\# of transistors) } & 17 & 13.5 & $-20.59 \%$ \\
\hline \multirow{3}{*}{ Setup Time } & Rise (ps) & -9.21 & -12.51 & $-35.83 \%$ \\
\cline { 2 - 5 } & Fall (ps) & 28.14 & 20.99 & $-25.41 \%$ \\
\cline { 2 - 5 } & Average (ps) & 9.47 & 4.24 & $-55.20 \%$ \\
\hline \multirow{3}{*}{ Hold Time } & Rise (ps) & 19.65 & 11.96 & $-39.13 \%$ \\
\cline { 2 - 5 } & Fall(ps) & 16.60 & 17.15 & $3.31 \%$ \\
\cline { 2 - 5 } & Average (ps) & 18.13 & 14.56 & $-19.70 \%$ \\
\hline \multirow{3}{*}{ Clock To Q Delay } & Rise (ps) & 131.11 & 89.95 & $-31.39 \%$ \\
\cline { 2 - 5 } & Fall (ps) & 121.01 & 145.97 & $20.63 \%$ \\
\cline { 2 - 5 } & Average (ps) & 126.06 & 117.96 & $-6.43 \%$ \\
\hline \multicolumn{2}{|c|}{ Leakage Power(nW) } & 0.61 & 0.33 & $-45.53 \%$ \\
\hline
\end{tabular}

For instance, Fig. 7 shows a TIG SiNWFET flip-flop with AND gate embedded in the first stage. In the TIG SiNWFET implementation, the first stage is a clocked AND gate, derived from [9]. The following stages remain unchanged as compared to Fig. 4. Note that the clock signal in the first stage is wired to the low-leakage controllability gate, leading to a larger power efficiency as a trade-off in internal delay.

Electrical simulations, depicted in Fig. 8, validates the AND/TSPC flip-flop functionality. Indeed, it shows that the output $\mathrm{Q}$ equals to $\mathrm{A} A N D \mathrm{~B}$ at each clock rising edge. Finally, we compare the performance between CMOS design in Fig. 3 and TIG SiNWFET design in Fig. 7. Table II shows that the area shrinks by $21 \%$, while the delay reduces by $6 \%$ on average and leakage power drops by $45 \%$. Leakage gain is accounted for power-efficiency of the TIG AND gate [9]. Nevertheless, note that the timing performance gain is lowered because of the use of the low-leakage controllability gate of TIG FETs.

\section{CONCLUSION}

In this paper, TSPC flip-flop circuit designs, leveraging the compactness offered by TIG SiNWFETs, are proposed. Experimental results, obtained by electrical characterization, show that TIG SiNWFET implementation improves area, delay and leakage power by nearly $20 \%, 30 \%$ and $7 \%$ respectively compared to CMOS design. In addition, we show that an AND gate can be embedded into the structure. This leads to an area reduction of $21 \%$, a delay reduction of $6 \%$ and a leakage reduction of $45 \%$ on average.

\section{ACKNOWLEDGMENT}

This work has been partially supported by the ERC senior grant NanoSys ERC-2009-AdG-246810.

\section{REFERENCES}

[1] C. Teh, T. Fujita, H. Hara, and M. Hamada, A 77\% energy-saving 22-transistor single-phase-clocking D-flip-flop with adaptive-coupling configuration in 40nm CMOS, IEEE International Solid-State Circuits Conference (ISSCC), pp. 338-340, Feb. 2011.

[2] J. M. Rabaey, A. Chandrakasan, and B. Nikolic, Digital Integrated Circuits, Second Edition, Prentice-Hall Publisher, 2002.

[3] Y. Ji-ren and C. Svensson, New Single-Clock CMOS Latches and Flip Flops Improved Speed and Power Savings, IEEE Journal Solid-State Circuits, Vol. 32, No. 1, pp. 62-69, Jan. 1997.

[4] S. Nomura, et al., A 9.7mW AAC-Decoding, 620mW H.264 720p 60fps Decoding, 8-Core Media Processor with Embedded Forward-BodyBiasing and Power-Gating Circuit in 65nm CMOS Technology, ISSCC Dig. Tech. Papers, pp. 262-264, Feb. 2008.

[5] Y. Lin et al., High-Performance Carbon Nanotube Field-Effect Transistor with Tunable Polarities, IEEE Transaction Nanotechnology, Vol. 4, No. 5, pp. 481-489, 2005.

[6] N. Harada et al., A polarity-controllable graphene inverter, Applied Physics Letters, Vol. 96, No. 1, pp. 012102 - 012102-3, 2010.

[7] M. De Marchi et al., Polarity Control in Double-Gate, Gate-All-Around Vertically Stacked Silicon Nanowire FETs, International Electron Devices Meeting (IEDM), San Francisco, California, USA, pp. 841-844, 2012.

[8] H. Iwai, Technology Roadmap for 22nm and Beyond, International Workshop on Electron Devices and Semiconductor Technology, pp. 1-4, 2009.

[9] J. Zhang, P.-E. Gaillardon, and G. De Micheli, Dual-Threshold-Voltage Configurable Circuits with Three-Independent-Gate Silicon Nanowire FETs, IEEE International Symposium on Circuits and Systems (ISCAS), Beijing, China, pp. 2111-2114, 2013.

[10] S. Bobba, M. De Marchi, Y. Leblebici and G. De Micheli, Physical Synthesis onto Sea-of-Tiles with Double-Gate Silicon Nanowire Transistors, 49th Design Automation Conference (DAC), San Francisco, California, USA, pp. 42-47, 2012.

[11] Cadence, Encounter Library Characterize User Guide, Product Version 8.1.3, September 2009.

[12] Predictive technology model. [Online]. Available: http://ptm.asu.edu

[13] P. Larson and C. Svensson, Impact of Clock Slope on True Single Phase Clocked (TSPC) CMOS Circuits, IEEE Journal Solid-State Circuits, Vol. 29, No. 6, pp. 723-726, Jun. 1994.

[14] N. Golinescu, A $2.5 \mathrm{~Gb} / \mathrm{s}$ CMOS Add - Drop Multiplexer for ATM, Master Thesis, University of Toronto, 1999. 